\title{
HARMONIC ANALYSIS AND THE GEOMETRY OF SUBSETS OF $\mathrm{R}^{n}$
}

\author{
GUY DAVID AND STEPHEN SEMMES
}

This subject has several natural points of view, but we shall start with the one that corresponds to the following question: Is there something like LittlewoodPaley theory which is uscful for analyzing the geometry of subsets of $\mathbf{R}^{n}$, in much the same way that traditional Littlewood-Paley theory is good for analyzing functions and operators? To explain what we mean by this let us first give examples of classical ideas of which we would like to have counterparts in this geometrical setting.

Let $\psi(x)$ be a smooth function on $\mathrm{R}^{n}$ with compact support, $\int \psi=0$, and possibly more vanishing moments as well. Given a function $f$ on $\mathbf{R}^{n}$, we can obtain a lot of useful information about it from the size of $\psi_{t} * f(x)$, where $\psi_{t}(x)=t^{-n} \psi\left(\frac{x}{t}\right)$, at least if $\psi$ is sufficiently nondegenerate. Whether $\psi_{t} * f \rightarrow 0$ as $t \rightarrow 0$, and how fast, tells us about the smoothness of $f$, for instance.

There are also useful reproducing formulas for $f$ with $\psi_{t} * f$ arising as the cocfficients, e.g.,

$$
\begin{aligned}
f(x) & =c \int_{0}^{\infty} \psi_{t} * \psi_{t} * f(x) \frac{d t}{t} \\
& =c \int_{0}^{\infty} \int_{\mathrm{R}^{n}} \psi_{t}(x-u) \psi_{t} * f(u) d u \frac{d t}{t},
\end{aligned}
$$

which holds if $\psi$ is radial and not identically 0 . This allows us to express $f$ as a supcrposition of simpler objects, namely the functions $\psi_{t, u}=\psi_{t}(\cdot-u)$.

In searching for a version of these idcas which is useful for analyzing subsets of $\mathbf{R}^{n}$ we could try the following naive approach. Let $E$ be a closed $d$-dimensional set in $\mathrm{R}^{n}$, and let $\mu$ denote the restriction to $E$ of $d$-dimensional Hausdorff measure. (Throughout this paper $d$ is an integer, $1 \leq d<n$.) We could attempt to analyze $E$ by applying the usual techniques to $\mu$, viewed as a measure on $\mathbf{R}^{n}$. In other words, we could try to derive information about $E$ from $\psi_{\iota} * \mu$ and the reproducing formula

$$
\mu=c \int_{0}^{\infty} \psi_{t} * \psi_{t} * \mu \frac{d t}{t}=c \int_{0}^{\infty} \int_{\mathbb{R}^{n}} \psi_{t, y} \psi_{\ell} * \mu(y) d y \frac{d t}{t}
$$

The second author is partially supported by the U.S. National Science Foundation and the Alfred P. Sloan Foundation. 
Unfortunately, it is not at all clear how to do anything useful with these. For example, if we take $E$ to be a $d$-plane, which is the most basic $d$-dimensional set, then $\psi_{t} * \mu$ and this reproducing formula are not so simple. They do not reflect the geometry so well.

To get a better idea of what is wrong with this approach, and what we should do instead, we should be more precise about what we want. We would like to have a method of analysis that can detect if $E$ is a $d$-plane, and, more generally, measure smoothness of $E$. We'd like to be able to discern rectifiability properties of $E$, e.g., to be able to distinguish totally unrectifiable Cantor sets from sets which are subsets of a countable union of $d$-dimensional Lipschitz graphs.

In this context $d$-planes play much the same role that constants (or affine functions) do in the traditional setting. The smoothness of a set is closely related to how well it can be approximated by a $d$-plane, just as the smoothness of a function can be measured by how well it is approximated by constants or affine functions.

Here is one way that this manifests itseif in traditional Littlewood-Paley theory. Suppose that $\psi$ has vanishing first monents in addition to $\int \psi=0$, so that $\psi * A=0$ for all affine functions $A$. Given a function $f$, we have then that $\left|\psi_{t} * f\right|$ is controlled by how well $f$ is approximated by affine functions. When we tried the naive approach to analyzing sets by Littlewood-Paley theory this did not work, because we do not have $\psi_{t} * \mu \equiv 0$ if $E$ is a $d$-plane.

However, if we look at $\psi_{t} * \mu(x)$ only for $x \in E$, then there are lots of $\psi$ 's so that this will vanish whenever $E$ is a $d$-plane, any $\psi$ which is odd, for instance. There is still a serious problem with this, though: how do we recover information about $E$ from $\left.\psi_{t} * \mu\right|_{E}$ ? We no longer have a reproducing formula as before.

It turns out that there is a way to overcome this difficulty. Before describing our results we state a couple of easy definitions, postponing momentarily the more complicated ones.

We say that $E$ is a regular $d$-dimensional set if it is closed and there is a $C>0$ so that

$$
C^{-1} R^{d} \leq \mu(E \cap B(x, R)) \leq C R^{d}
$$

for all $x \in E, R>0$. This means in particular that $E$ is a space of homogeneous type (with measure $\mu$ and the Euclidean distance) in the sense of [CW].

A measure $\lambda$ on $E \times \mathbf{R}_{+}$is called a Carleson measure if there is a $K>0$ so that

$$
\lambda((E \cap B(x, R)) \times(0, R)) \leq K R^{d}
$$

for all $x \in E, R>0$. Such measures behave in some ways as though they are $d$-dimensional, even though they live on a $(d+1)$-dimensional set.

Theorem. Let $E$ be a regular d-dimensional sabset of $\mathbf{R}^{\mathbf{n}}$. The following 
are equivalent.

(A) For each odd, smooth, compactly supported function $\psi$ on $\mathbf{R}^{n}$,

$$
\sum_{j=-\infty}^{\infty}\left|\int_{E} 2^{-j d} \psi\left(\frac{x-y}{2^{j}}\right) d \mu(y)\right|^{2} d \mu(x) d \delta_{2-j}(t)
$$

is a Carleson measure on $E \times \mathbf{R}_{+}$.

(B) E admits a corona decomposition.

(C) E has big pieces of Lipschitz images of subsets of $\mathrm{R}^{d}$.

(D) E has very big pieces of bilipschitz images of subsets of $\mathbf{R}^{d}$.

(E) There is an $A_{I}$-weight $\omega$ on $\mathbf{R}^{d}$ and an $\omega$-regular mapping $z: \mathbf{R}^{d} \rightarrow$ $\mathbf{R}^{n+1}$ with $E \subseteq z\left(\mathbf{R}^{d}\right)$.

Let us give some explanations. In $(A) d \delta_{2^{-j}}(t)$ denotes the Dirac mass in $t$ at $2^{-j}$. One can think of (A) as a gcometric analogue of the fact that $f \in$ $\mathrm{BMO}\left(\mathbf{R}^{n}\right)$ if and only if $\left|\psi_{t} * f(x)\right|^{2} d x \frac{d t}{t}$ is a Carleson measure on $\mathbf{R}^{n} \times \mathbf{R}_{+}$ for any $\psi$ as above.

The definition of a corona decomposition is complicated and will be put off for a while. It is a geometric condition that concerns the approximation of $E$ by Lipschitz graphs.

The precise statement for $(C)$ is that there exist $K, \eta>0$ so that for each $x \in E, R>0$ there are subsets $F$ of $E \cap B(x, R)$ and $G$ of $\mathbf{R}^{d}$, and a map $\rho: G \rightarrow F$, such that $\rho(G)=F, \mu(F) \geq \eta R^{d}, G$ has diameter $\leq R$, and $\rho$ is Lipschitz with norm $K$, i.e.,

$$
|\rho(x)-\rho(y)| \leq K|x-y|
$$

for all $x, y \in G$. Of course you can extend $\rho$ to a Lipschitz mapping of $\mathbf{R}^{d}$ into $\mathbf{R}^{n}$.

(D) is much the same as (C) except for a change in quantifiers: for each $\varepsilon>0$ there is a $K>0$ so that for each $x \in E, R>0$ there exist $F, G, \rho$ as above except that now $\rho$ is bilipschitz, i.e.,

$$
K^{-1}|x-y| \leq|\rho(x)-\rho(y)| \leq K|x-y|,
$$

and

$$
\mu((E \cap B(x, R)) \backslash F) \leq \varepsilon R^{d},
$$

so that $\rho(G)$ covers $E \cap B(x, R)$ except for a small set. It turns out that you can extend $\rho$ to a bilipschitz mapping of $\mathbf{R}^{d}$ into $\mathbf{R}^{n}$ if $n \geq 2 d+1$. Of course (D) implies (C).

For (E) we begin by recalling that $\omega$ is an $A_{1}$ weight on $\mathbf{R}^{d}$ if there is a $C>0$ so that

$$
\frac{1}{|Q|} \int_{Q} \omega \leq C(e s s \inf \omega)
$$


for all cubes $Q$. It is well-known that this implies that $\omega$ is an $A_{\infty}$-weight, which is characterized by the condition

$$
\left(\frac{1}{|Q|} \int_{Q} \omega^{I+\delta}\right)^{\frac{1}{1+\delta}} \leq C \frac{1}{|Q|} \int_{Q} \omega
$$

for some $C, \delta>0$ and all cubes $Q$.

To say that $z: \mathbf{R}^{d} \rightarrow \mathbf{R}^{n+1}$ is an $\omega$-regular mapping means that $z$ has locally integrable distributional derivatives, $|\nabla z| \leq C \omega^{\frac{t}{d}}$ a.e., and

$$
\omega\left(\left\{x \in \mathbf{R}^{d}:|z(x)-y| \leq R\right\}\right) \leq C R^{d}
$$

for all $y \in \mathbf{R}^{n+1}, R>0$, where $\omega(A)=\int_{A} \omega(x) d x$. Here we require that $\omega$ be at least an $A_{\infty}$ weight, so that $|\nabla z| \in L_{\text {loc }}^{d+\varepsilon}$ for some $\varepsilon>0$. If we only required that $\omega$ be an $A_{\infty}$ weight instead of an $A_{1}$ weight, then the resulting variation $\left(E^{\prime}\right)$ of $(E)$ would still be equivalent to the other conditions.

If $n \geq 2 d$ we can even require that the mapping $z$ in (E) take values in $\mathrm{R}^{n}$. Notice that when $d=1$ we can always take $\omega=1$ by replacing $z$ by an arclength parameterization. Thus, when $n=2$ and $d=1$, (E) says that $E$ is contained in a regular curve.

Let us relate this theorem to more familiar issues in harmonic analysis, the behavior of singular integrals on $E$ in particular. It is not hard to show that (A) is equivalent to the square function estinate

$$
\sum_{j=-\infty}^{\infty} \int_{E}\left|\int_{E} 2^{-j d} \psi\left(\frac{x-y}{2^{j}}\right) f(y) d \mu(y)\right|^{2} d \mu(x) \leq C(\psi)\|f\|_{L^{2}(E)}^{2}
$$

for all $\psi$ as above and $f \in L^{2}(E)$. This equivalence is obtained using standard techniques, including those of the $T(1)$ variety. There is an important conccptual difference between $(A)$ and $\left(A^{\prime}\right)$, however; $\left(A^{\prime}\right)$ is an estimate for square function operators on $E$, while we think of $(A)$ as a squarc function estimate applied to $E$ itself (or, more precisely, to $\mu$ ).

Another equivalent condition is

$$
\sup _{\varepsilon>0} \int_{E}\left|\int_{\substack{y \in E \\|x-y|>\varepsilon}} K(x-y) f(y) d \mu(y)\right|^{2} d \mu(x) \leq C(K)\|f\|_{L^{2}(E)}^{2}
$$

for all $f \in L^{2}(E)$ and all smooth, odd functions $K$ on $\mathbf{R}^{n} \backslash\{0\}$ which satisfy $\left|\nabla^{j} K(x)\right| \leq C(j)|x|^{-d-j}$ for $j=0,1,2, \ldots$. Indeed, one can prove that $(\alpha)$ implies $\left(A^{\prime}\right)$ using a standard trick with Rademacher functions, while (C), (D), 
(E), and (E') all imply ( $\alpha$ ), by the results of [D1]. (For (C) we also need the main result in [J3].) One can also show directly that (B) implies $(\alpha)$, as in $[\mathrm{Se}]$.

Interesting particular examples of such kernels $K(x)$ are the Cauchy kernel $K(z)=\frac{1}{z}$ on $\mathbf{C}$ and its counterpart in $\mathbf{R}^{n}, K^{\prime}(x)=\frac{x}{|x|^{n}}(d=n-1)$. The doublelayer potential can of course be expressed in terms of this second example. We do not know if the condition you get by demanding that $(\alpha)$ hold only for $K(x)=\frac{x}{|x|^{d+1}}$ is still equivalent to the others.

There is also a condition which is a version of Peter Jones' geometric lemma and which is equivalent to the others. Given $x \in E$ and $t>0$ set

$$
\beta(x, t)=\inf _{P} \frac{1}{t^{d}} \int_{B(x, t) \cap E} \frac{\operatorname{dist}(y, P)}{t} d \mu(y),
$$

where the infimum is taken over all $d$-planes $P$. Thus $\beta(x, t)$ measures the extent to which $E$ can be approximated by a $d$-plane. Jones worked with a sirnilar quantity when $d=1$, but with the $L^{3}$ norm replaced by an $L^{\infty}$ norm.

The conditions above are also equivalent to (F) $\beta(x, t)^{2} d \mu(x) \frac{d t}{t}$ is a Carleson measure on $E \times \mathbf{R}_{+}$.

Jones and his student Fang have shown that this can fail in higher dimensions even for Lipschitz graphs if we define $\beta(x, t)$ in terms of an $L^{\infty}$ norm instead of an $L^{1}$ norm. We would, however, still have an equivalent condition if we replaced the $L^{1}$ norm by an $L^{p}$ norm, $p<\frac{2 d}{d-2}$.

The equivalence of $(F)$ with $(E)$ (for regular sets) can be viewed as a higherdimensional version of the results of Jones [J2] characterizing the 1-dimensional scts which are subsets of rectifiable curves in terms of quadratic estimates on the analogue of $\beta(x, t)$ defined in terms of an $L^{\infty}$ norm. Jones' techniques do not work when $d>1$, but they also do not require the set to be regular.

Conditions $(A)$ and $(F)$ are very similar in some ways. In particular, each is a square function condition on $E$ that has a classical counterpart for functions on $\mathbf{R}^{n}$. For $(F)$ that counterpart is the following. Let $f$ be a locally integrable function on $\mathbf{R}^{d}$. Set

$$
\gamma(x, t)=\inf _{a} \frac{1}{t^{d}} \int_{B(x, t)}|f(x)-a(x)| d x,
$$

where the infimum is taken over all affine functions $a$. Then

$$
\int_{\mathbf{R}^{d}} \int_{0}^{\infty} \gamma(x, t)^{2} \frac{d t}{t^{3}} d x \approx \int|\nabla f|^{2} .
$$

(See [Do], for instance.) This is a well-known cousin of the more farnilar square function estimate in which $\gamma(x, t)$ is replaced by a second difference (which also 
measures how well $f$ can be approximated by affine functions). Notice that the fact that (F) holds for Lipschitz graphs can be derived immediately from this estimate. Indeed, if $\Gamma$ is the graph of a Lipschitz function $A$, it is easy to pass back and forth between the $\beta(x, t)$ 's for $\Gamma$ and the $\gamma(x, t)$ 's for $A$.

This theorem telis us a lot about the relationship between the analysis and the geometry on (regular) sets in $\mathbf{R}^{n}$, but this relationship is very complicated. For example, the equivalence between $\left(A^{\prime}\right)$ and $(\alpha)$ is a purely analytical statement, but we don't know how to prove that $\left(A^{\prime}\right)$ implies $(\alpha)$ without going through the geometry, and it is not clear if we should be able to do this directly. Similarly, it is not clear how to prove that, say, (D) implies (E) without using singular integral operators.

Thus the singular integral operators provide a useful tool for dealing with the geometrical issues, because they allow you to transform certain kinds of geometrical information into different geometrical information (to wit, the corona decomposition). Another example of this occurred in [Se].

Some of the geometrical issues that we've discussed also arise in geometric measure theory, at least in a qualitative form, and it is very helpful to compare the two points of view, and so we now give a brief overview of some aspects of geometric measure theory.

Let $H^{d}$ denote $d$-dimensional Hausdonff measurc. Let $A$ be a subset of $\mathbf{R}^{n}$ with Hausdorff dimension $d$. We say that $A$ is rectifiable if there is a countable family of Lipschitz mappings $f_{i}: \mathbf{R}^{d} \rightarrow \mathbf{R}^{n}, i=1,2, \ldots$, such that $H^{d}(A \backslash$ $\left.\left(\cup f_{i}\left(\mathbf{R}^{d}\right)\right)\right)=0$. In other words, $A$ is covered by the union of the images of the $f_{i}$ 's except for a set of measure zero. This is equivalent to requiring that $A$ be covered (except for a set of measure zero) by a countable union of Lipschitz graphs, or even $C^{1}$ submanifolds.

We say that $A$ is unrectifiable if $H^{d}(A \cap B)=0$ for every rectifiable set $B$. A standard example of this (for $d=1, n=2$ ) is obtained as follows. First take the: Cantor set contained in $[0,1]$ built from the usual construction except that yeu remow: the middle $\frac{1}{2}$ of each interval at each stage. The Cartesian product of this set with itself has finite, positive 1-dimensional Hausdorff measure, but it is unrectifiable.

(Jie es the basic results is that if $A$ is a set with $H^{d}(A)<\infty$, then $A$ has a rectifinde: subse: $B$ such thint $A \backslash B$ is unrectifiable.

Clearly comditions (C), (D), (E), and (E') above are closely related to rectifintsility. Centiminly $E$ is rectifiable if it satisfies onc of those conditions, as one can tasily shesw, but the converse is net true. Rectifiability is really a qualitative: utotion, whilo condlitions (C) ( $E^{\prime}$ ) are cquantitative. Like so many things in harmonic analysis, (C) (E') are "uniform over all scales"; they are not changed by translating or clikting the set. The relationship between rectifiability and (C) $\left(E^{\prime}\right)$ is a ket like the one betwen measurable functions that are positive a.e. ankl $A_{x}$ weights.

One of the isstey addressed in geometric measure theory is the problem of 
finding geometrical characterizations (or criteria) for rectifiability and unrectifability. One characterization is given in terms of the existence or nonexistence of approximate tangent planes. One can think of this as being analoguous to the existence of derivatives in the $L^{p}$ sense, say, of a function on $\mathbf{R}^{n}$, which suggests that one look for associated square functions: just as differentiability properties of functions on $\mathbf{R}^{n}$ can be characterized by square functions (as in [St]), there ought to be square function characterizations of rectifiability. Although the results of Jones [J2] and the equivalences above go a long way toward dealing with this issue, the role of square functions in these geometric problems is far from being fully understood.

Another characterization of rectifiability goes as follows. Let $A$ be an $H^{d}$. measurable set with $H^{d}(A)<\infty$. Then $A$ is rectifiable if and only if for every (measurable) subset $B$ of $A$ with $H^{d}(B)>0$ we have $H^{d}\left(P_{V}(B)\right)>0$ for almost all $d$-planes $V$, where $P_{V}$ denotes the orthogonal projection onto $V$. (The notion of "almost every $d$-plane" makes sense because the set of $d$-planes is a smooth manifold.) Similarly $A$ is unrectifiable if $H^{d}\left(P_{V} A\right)=0$ for almost all $Y$.

We would very much like to have a version of this last result with estimates. That is, we would like to know whether a regular set that has (in some sense) big projections on a lot of $d$-planes uniformly on all scales has to have big pieces of Lipschitz graphs, say. Unfortunately the techniques tased in geometric measure theory do not provide such quantitative results.

This is really just one example of the general problem of finding quantitative versions of qualitative results in geometric measure theory, if they exist. Here are two others.

Suppose you have a Lipschitz function $f$ defined on the unit cube $Q$ in $\mathbf{R}^{n}$ and taking values in $\mathbf{R}^{n}$. It is a classical fact that if $f(Q)$ has positive measure, then there is a set $A \subseteq Q$ with positive measure such that $\left.f\right|_{A}$ is bilipschitz. A quantitative version of this was proved in [D2]: if $f$ has Lipschitz norm $\leq 1$ and $|f(Q)| \geq \delta$, then there exists $E \subseteq Q$ with $|E| \geq \varepsilon$ such that

$$
|f(x)-f(y)| \geq \varepsilon|x-y| \text { for } x, y \in E
$$

where $\varepsilon$ depends only on $n$ and $\delta$. The proof used a complicated stopping time argument. Peter Jones [J3] later gave a much simpler proof in which square function estimates played a crucial role.

If we replace (C) by the requirement that $E$ have big pieces of Lipschitz graphs, do we get another equivalent condition? Qualitative versions of this are given by some of the characterizations of rectifiability which we mentioned when we stated the definition of rectifiability.

References for the topics in geometric measure theory that we've discussed include $[\mathrm{M}],[\mathrm{Si}]$, and $[\mathrm{F}]$. Chapter 6 in $[\mathrm{M}]$ is a particularly good place to start. 
In the remainder of this article we explain the definition of a corona decomposition and also sketch the proof of (A) implies (B), which is the heart of the theorem.

Roughly speaking, to say that $E$ admits a corona decomposition means that $E \times \mathbf{R}_{+}$can be decomposed into two regions, the good part and the bad part, with the following properties. The bad part is small, in the sense that it is controlled by a Carleson measure. The good part can be decomposed into a family of stopping-time regions, on each of which $E$ is well-approximated by a Lipschitz graph with small constant. There aren't too many of these stopping time regions, in the sense that they satisfy a Carleson measure packing condition.

To make this precise it is helpful to use a farnily of subsets $\Delta$ of $E$ which plays the same role as dyadic cubes in $\mathbf{R}^{d}$ do. Namely, there is a $C>0$ so that:

$$
\Delta=\bigcup_{j=-\infty}^{\infty} \Delta(j), \text { where each } \Delta(j) \text { is a partition of } E ;
$$

(4) if $Q_{1} \in \Delta(j), Q_{2} \in \Delta(k), j \leq k$, then either $Q_{1} \subseteq Q_{2}$ or $Q_{1} \cap Q_{2}=\emptyset$;

(5) if $Q \in \Delta(j)$, then $C^{-1} 2^{j} \leq \operatorname{diam} Q \leq C 2^{j}$ and $C^{-1} 2^{j d} \leq \mu(Q) \leq C 2^{j d}$.

One can even build the cubes so that they have small boundary in a certain sense. (See [D2].) This property is very useful in passing from "dyadic" analysis to "ordinary" analaysis on $E$. (An example of this occurred in [Se].)

In defining a corona decomposition it is better to work with $\Delta$ instead of $E \times \mathrm{R}_{+}$. We say that $E$ admits a corona decomposition if for each $\eta>0$ we can partition $\delta$ into the good set $\mathcal{G}$ and the bad set $\mathcal{B}$ with the following propertics.

The bad set is not too large, in that it satisfies a Carleson measure packing conditon,

$$
\sum_{\substack{Q \in \mathcal{B} \\ \mathcal{R} \subseteq R}}|Q| \leq C|R| \quad \text { for all } R \in \Delta
$$

The good set $\mathcal{G}$ can be partitioned into a family $\mathcal{F}$ of subsets $S$ of $\mathcal{G}$, such that:

(7) each $S$ is coherent, which means that it has a maximal element $Q(S)$, and that if $Q \in S, Q^{\prime} \in \Delta, Q \subseteq Q^{\prime} \subseteq Q(S)$, then $Q \in S$;

(8) for each $S$ there is a $d$-plane $P$ and a Lipschitz function $A: P \rightarrow P^{\perp}$, $\|\nabla A\|_{\infty} \leq \eta$, whose graph $\Gamma=\{p+A(p): p \in P\}$ approximates $E$ wcll from the viewpoint of $S$, in the sense that

$$
\operatorname{dist}(x, \Gamma) \leq \eta \operatorname{diam} Q
$$


whenever $x \in E, \operatorname{dist}(x, Q) \leq \operatorname{diam} Q$;

(9) there aren't too many of the $S$ 's, in that they satisfy the packing condition

$$
\sum_{\substack{S \in \mathcal{F} \\ Q(S) \subseteq R}}|Q(S)| \leq C|R| \text { for all } R \in \Delta .
$$

Such a decomposition is of course very close in spirit to the classical corona construction of Carleson; he was building stopping-time regions (in the upper half-plane, say) on which a given harmonic function is almost constant, while we want stopping-time regions on which the geometry of $E$ is almost constant.

There are a number of places where something like a corona decomposition has been used before. One is in the work of Garnett and Jones [GJ] on the corona problem on Denjoy domains. Although their decomposition is rather different at the level of details, it is very similar in spirit. Another is in a proof of Peter Jones on the boundedness of the Cauchy intcgral on regular curves [J1], and also in his estimates for his version of the $\beta$ 's (2) for rectifiabie curves [J2]. A corona decomposition also showed up in [Se] for a certain class of hypersurfaces in $\mathbf{R}^{n}$, in connection with square function estimates for the Cauchy kernel.

In each of these cases something like a corona decomposition was obtained by applying the corona construction to a function that somehow controlled the geometry. In our case we cannot apply the corona construction so directly, but we shall use many of the same ideas.

The notion of a corona decomposition turns out to be very useful despite its technical nature. It acts as a bridge between (A) and (F) on the onc hand and (C), (D), and (E) on the other; that is, in proving the theorem we show that both (A) and (F) imply (B), and that (B) implies each of (C), (D), and (E).

We now try to give a rough sketch of the sort of things that show up in the proof that (A) implies the existence of a corona decomposition.

The first step is to prove that (A) implics a weak form of (F). Given $k>0$ and $Q \in \triangle$, set

$$
\beta_{\infty}(Q)=\inf _{P} \sup _{x \in k Q} \frac{\operatorname{dist}(x, P)}{\operatorname{diam} Q},
$$

where the infirmum is taken over all $d$-plancs $P$, and $k Q=\{x \in E: \operatorname{dist}(x, Q) \leq$ $(k-1) \operatorname{diam} Q\}$. We say that $E$ satisfies the weak geometric lemma (WGL) if for each $\varepsilon>0$,

$$
\sum_{\substack{Q \subseteq R \\ \beta_{\infty}(\bar{Q}) \geq \varepsilon}}|Q| \leq C(\varepsilon)|R| \text { for all } R \in \Delta .
$$

In other words, the cubes for which $\beta_{\infty}(Q) \geq \varepsilon$ satisfy a packing condition. 
The proof that (A) implies (WGL) is not terribly complicated, but it is somewhat tricky and does not lend itself well to brief exposition.

The proof that (A) + (WGL) implies (B) is much more complicated, but the basic idea is fairly simple. Fix $\eta>0, \eta$ small. We need to split $\Delta$ into $\mathcal{B} \cup \mathcal{G}$ as above. Let $\varepsilon, \delta>0$ be small and positive, and $k>0$ be large, to be chosen later; $k$ is not allowed to depend on $\varepsilon, \delta$, and $\varepsilon$ will be much smaller that $\delta$. We put all the $Q$ 's with $\beta_{\infty}(Q) \geq \varepsilon$ into $\mathcal{B}$, but we shall put other cubes into $\mathcal{B}$ as well. Of course we can put anything we want into $B$, as long as we maintain (6).

If $Q \notin \mathcal{B}$, so that $\beta_{\infty}(Q)<\varepsilon$, let $P_{Q}$ be a good $d$-plane for $Q$. It is not hard to show that two candidates for $P_{Q}$ have to be close together, using the regularity of $E$.

It is also not difficult to show that we can enlarge $\mathcal{B}$ in such a way that (6) still holds and such that $\Delta \backslash \mathcal{B}$ can be partitioned into a family $\mathcal{F}$ of sets $S$ of cubes such that $(7)$ holds, and also such that

(12) if $Q \in S$, then angle $\left(P_{Q}, P_{Q(S)}\right) \leq \delta$ and

(13) if $Q$ is a minimal element of $S$, then either $Q$ has a son in $B$, or angle $\left(P_{Q}, P_{Q(S)}\right) \geq \delta / 2$.

In other words, in building $S$ we keep going down and we don't stop unless we run into a bad cube, or unless $P_{Q}$ has turned from $P_{Q(S)}$ by a definite amount.

To show that $E$ admits a corona decomposition we have to prove that each $S$ satisfies (8) and (9). The first is obtained fairly easily using the fact that each $Q$ is well-approximated by $P_{Q}$ and the $P_{Q}$ 's don't turn too much. In fact you can build $A, \Gamma$ so that $\|\nabla A\|_{\infty} \leq C \delta$ and

$$
\sup _{x \in(k / 2) Q} \operatorname{dist}(x, \Gamma) \leq C \varepsilon \operatorname{diam} Q .
$$

Thus $\Gamma$ approximates $E$ to $\operatorname{ordcr} \varepsilon$, not just $\delta$, which turns out to be very important.

Proving (9) is much more difficult. There is a simple preliminary reduction which is helpful: we need only consider the $S$ 's for which

$$
\frac{1}{2}|Q(S)| \leq \mu(\cup\{Q: Q \text { is a minimal element in } S \text { such that angle }
$$

$$
\left.\left.\left(P_{Q}, P_{Q(S)}\right) \geq \delta / 2\right\}\right)
$$

i.e., for which there are plenty of this kind of minimal cube in $S$.

There is a sccond preliminary reduction that we use, but it requires more notation. Define $d(x)$ on $\mathbf{R}^{n}$ by

$$
d(x)=\inf _{Q \in S}\{\operatorname{dist}(x, Q)+\operatorname{diam} Q\} .
$$


Define the "summing region" $\sigma \subseteq E \times \mathbf{Z}$ by

$$
\sigma=\left\{(x, l): x \in k_{0} Q(S), \gamma_{0} d(x) \leq 2^{l} \leq \operatorname{diam} Q(S)\right\},
$$

where $k_{0}>0$ (large) and $\gamma_{0}>0$ (small) are chosen later, independently of $\varepsilon$ and $\delta$, but with $k_{0}$ much smailer than $k$. Given $\psi$ as in (A), define $J=J(S, \psi)$ by

$$
J(S, \psi)=\int_{(x, l) \in \sigma}\left|\int_{E} \psi_{l}(x-y) d \mu(y)\right|^{2} d \mu(x),
$$

where $\psi_{l}(x)=2^{-l d} \psi\left(\frac{x}{2^{2}}\right)$. To prove (9) it suffices to show that there is a finite family $\Psi$ of $\psi$ 's so that

$$
\sum_{\psi \in \Psi} J(S, \psi) \leq \tau|Q| \text { implies that } S \text { does not satisfy (15), }
$$

at least if $k_{0}^{-1}, \gamma_{0}$, and $\varepsilon$ are small enough. Here $\tau>0$ and $\Psi$ are allowed to depend on any of the constants but not on $S$. This reduction is not hard to obtain from the previous one and (A).

The proof of (16) is sort of technical, but the rough idea is not too bad. We first want to show that $J(\psi, S)$ being small implies certain square function estimates on $A$, which in turn imply that $\nabla A$ has small $L^{2}$ oscillation, small compared to $\delta$. Once we've done that it is not hard to show that $S$ can't satisfy (15).

The idea for passing from a bound on $J(S, \psi)$ to a square function estimate on $A$ is that $E$ is well-approximated by $\Gamma$, and so if we replace $E$ by $\Gamma$ in $J(S, \psi)$, replace the $d \mu$ 's by something more pleasant, and choose $\psi$ correctly, we should get some sort of square function quantity of $A$, while only incurring errors that are small compared to $\delta$. Replacing $E$ by $\Gamma$ is not so hard, using (14), and we get errors of order $\varepsilon$, which is fine. Dealing with the $d \mu$ 's is more complicated, but doable, and choosing the $\psi$ 's correctly is easy.

The preceeding outline of the argument is very sketchy, but hopefully it gives some idea of what goes on.

It is interesting to look at the precceding argument from the point of view of nonlinear analysis in general. When faced with a nonlinear problem --- such as proving that (A) implies (B) - it is natural to look first at small perturbations of a simple case, which you can try to treat by reducing to a linearized problem. For example, in our situation we could look at small perturbations of $d$-planes, such as Lipschitz graphs with small constants. Usually it is a serious problem to get from small perturbations to the general case. This is precisely what the corona construction did for us.

It is also interesting to compare the corona decomposition with more traditional ideas in Littlewood-Paley theory. In some sense the corona decomposition tells us how to break up $E$ into simpler pieces with estimates that allow 
us to maintain control, in much the same way that Littlewood-Paley theory permits you to break up a function into simpler pieces with good estimates, using the reproducing formula (1), for instance. The work of Carleson provides alternative methods for expressing functions as superpositions of simpler objects, e.g., his construction for writing a BMO function as the sum of a bounded function and the balayage of a Carleson measure. Although such constructions are more complicated and nonlinear than the elegant reproducing formulas, they seem to be more well-suited to our more geometrical context. One reason for this is that we do not have a good geometrical counterpart to the notion of orthogonality in function spaces.

\section{References}

[CW] R.R. COIFMAN AND G. WEIss, Extensions of Hardy spaces and their uses in analysis, Bull. Amer. Math. Soc. 83 (1977), 569-645.

[D1] G. DAvID, Opérateurs d'intégrale singulière sur les surfaces régulières, Ann. Sci. Ec. Norm. Sup. (4) 21 (1988), 225-258.

[D2] G. DAvID, Morceaux de graphes Lipschitziens et integrales singulières sur une surface, Rev. Mat. Iberoamericana 4 (1988), 73-114.

[DJ] G. DAVID AND D. JERISON, Lipschitz approximations to hypersurfaces, harmonic measure, and singular integrals, Indiana Math. J. (to appear).

[Do] J.R. Donronsoro, A characterization of potential spaces, Proc. Amer. Math. Soc. 95 (1985), 21-31.

[F] H. Frderen, "Geometric Measure Theory," Springer-Verlag, 1969.

[Ga] J.B. Gannetr, "Bounded Analytic Functions," Academic Press, 1981.

[G.J] J.B. GAGNETT AND P.W. JoNES, The corona theorem for Denjoy domains, Acta. Math. 155 (1985), 29-40.

[J1] P.W. JONES, Square functions, Cauchy integrals, analytic capacity, and harmonic measure, Harmonic Analysis and partial differential equations, Proccedings of the conference held at El Escorial, Spain (June, 1987), edited by J. García-Cuerva, Lecture Notes in Math. 1384, Springer-Verlag, 1989 , pp. $24-68$.

[J2] P.W. JoNEs, Rectifiable sets and the travelling salesman problem, preprint.

[J3] P.W. Jones, Lipschitz and bi-Lipschitz functions, Rev. Mat. Iberoamericana 4 (1988), 115-122.

[M] P. Mat'Tila, "Lectute Notes on Geometric Measure Theory," Departamento de Matemáticus, Universidad de Extremadura, 1986.

[Se] S. SEmmes, Geometry vs analysis on a class of rectifiable hypersurfaces in $\mathbf{R}^{n}$, Indiana Math. J. (to appear). 
[Si] L. Simon, "Lectures on Geometric Measure Theory," Proc. of the Centre for Mathematical Analysis 3, Australian National University, 1983.

[St] E. STEIN, "Singular Integrals and Differentiability Properties of Functions," Princeton University Press, 1970.

Guy David: Université de Paris Sud 91405 Orsay

FRANCE

Stephen Semmes: Rice University

Houston TX 77251

U.S.A. 